\title{
Correction: The pleiotropic effects of TNFa in breast cancer subtypes is regulated by TNFAIP3/A20
}

\author{
Eunmi Lee ${ }^{1} \cdot$ Maria Ouzounova, ${ }^{1,6} \cdot$ Raziye Piranlioglu $^{1} \cdot$ Minh Thu Ma ${ }^{1} \cdot$ Mustafa Guzel $^{2} \cdot$ Daniela Marasco $^{3}$. \\ Ahmed Chadli ${ }^{1}$ - Jason E. Gestwicki ${ }^{4}$ - John K. Cowell ${ }^{1} \cdot$ Max S. Wicha ${ }^{5} \cdot$ Khaled A. Hassan $^{4} \cdot$ Hasan Korkaya $\mathbb{D}^{1}$
}

Published online: 13 June 2019

(c) The Author(s), under exclusive licence to Springer Nature Limited 2019

\section{Correction to: Oncogene 38: 469-482}

https://doi.org/10.1038/s41388-018-0472-0

Published online 30 August 2018

Following publication of this article the authors noted that the Acknowledgments section had been omitted. This section should read as follows:
Acknowledgements We gratefully acknowledge the generous help from Flow Cytometry, Genomics Core facilities and Laboratory of Animal Services. This work was supported by startup funds to H.K. by Georgia Cancer Center. Additional research fundings to H.K. provided by American Cancer Society Institutional fund, Augusta University Research Inc. Bridge funding Forbes Institute research fund and R35CA197585 funding is to M.S.W.
Hasan Korkaya

hkorkaya@augusta.edu

1 Department of Biochemistry and Molecular Biology, Georgia Cancer Center, Augusta University, Augusta, GA 30912, USA

2 Regenerative and Restorative Research Center (REMER), Medipol University, Istanbul, Turkey

3 Department of Pharmacy, University of Naples Federico II, 80134 Naples, Italy

4 University of California, San Francisco, USA

5 Comprehensive Cancer Center, University of Michigan, Ann Arbor, MI 48109, USA

6 Present address: Department of Cancer Cell Plasticity, Cancer Research Center of Lyon (CRCL), Lyon, France 